\title{
Qualité nutritionnelle et bactériologique des échantillons de quelques produits laitiers locaux de la chaîne de production au Burkina Faso
}

\author{
Vinsoun MILLOGO*, Mariétou SISSAO et Georges Anicet OUÉDRAOGO
}

Laboratoire de Recherche et d'Enseignement en Santé et Biotechnologies Animales, Institut du Développement Rural, Université Nazi Boni de Bobo-Dioulasso, 01 BP 1091 Bobo-Dioulasso, 01 Burkina Faso.

*Auteur correspondant, E-mail: paravins@yahoo.fr; Tel (+226)70228995.

\section{REMERCIEMENTS}

Les auteurs de cet article adressent leurs sincères remerciements au Conseil Ouest et Centre Africain pour la Recherche et le Développement Agricole (CORAF) pour avoir financé cette étude à travers le projet " Appui à l'amélioration durable de la productivité et de la compétitivité des filières laitières bovines en Afrique de l'Ouest et du Centre » (AMPROLAIT).

\section{RESUME}

L'un des freins au développement de la filière laitière en Afrique de l'Ouest et du Centre est l'insuffisance de compétitivité des produits laitiers locaux face à ceux importés. L'objectif de cette étude est de déterminer les paramètres à risque et la valeur nutritive des laits. Un échantillonnage des laits crus, pasteurisés et yaourts fabriqués localement a été réalisé au niveau des fermes, des collecteurs, des vendeuses ou vendeurs, des laiteries et des supermarchés à Bobo-Dioulasso (Burkina Faso). Les échantillons ont été analysés pour leur composition chimique et les cultures ont été faites pour isoler Escherichia Coli, la flore aérobie mésophile totale, Staphylococcus aureus et les Streptocoques. Les analyses statistiques ont été effectuées par la méthode ANOVA (analyse des variances). Le pourcentage de matières grasses (MG), de matières protéiques (MP), de lactose $(\mathrm{L})$, de matière sèche (MS) et de de matières minérales $(\mathrm{MM})$ reflètent les valeurs d'un lait de bonne valeur nutritive pour chaque maillon excepté les laits des vendeuses $(1,3 \pm 0,8 \% \mathrm{MG}, 1,3 \pm 0,6 \% \mathrm{MP}, 2,2 \pm 1,0 \%$ $\mathrm{L}, 5,1 \pm 2,7 \% \mathrm{MS}$ et de $3,8 \pm 1,9 \% \mathrm{MM})$. On peut conclure que l'hygiène des laits constitue un réel problème et nécessite un effort supplémentaire des acteurs de la filière.

(c) 2018 International Formulae Group. All rights reserved.

Mots clés: Echantillonnage, maillons de la chaîne de production, laits, composition, hygiène, kits.

\section{Nutritional and bacteriological quality of some local dairy products samples of Burkina Faso' production chain}

\begin{abstract}
The step down development of the dairy chain value in West and Central Africa is the weak competiveness of local dairy products compared with import products. The aim of the study was to carry out milk sampling in order to determine the main risk criteria for hygiene and nutritive value of dairy products. A sampling of raw and pasteurized milks, yogurts made in Burkina Faso was carried out at farm level, from milk
\end{abstract}


collectors, at local selling point, at processing unit level and at supermarkets level in Bobo-Dioulasso (Burkina Faso). The samples were run for their composition and Escherichia coli, total mesophilic aerobic flora, Staphylococcus aureus and Streptococcus sp were isolated. Analysis of variance (ANOVA) was applied as statistic method. The results showed that milk fat, protein, lactose, dry matter and solids non fat (SNF) contents meet the requirements for nutritive value of raw milks and dairy products along the dairy chain value. However, raw milk from local selling points showed very bad milk composition $(1,3 \pm 0,8 \%$ fat, $1,3 \pm 0,6 \%$ Protein, $2,2 \pm 1,0 \%$ Lactose, $5,1 \pm 2,7 \%$ dry matter, $3,8 \pm 1,9 \% \mathrm{SNF})$. It was concluded that milk hygiene remains a serious challenge for competitiveness of local dairy products in Burkina Faso.

(C) 2018 International Formulae Group. All rights reserved.

Keywords: Sampling, dairy value chain, milks, composition, hygiene, hygiene tools.

\section{INTRODUCTION}

Le lait fait partie des aliments les plus exigeants en termes de qualité et d'hygiène. Tout ceci est lié à sa richesse en nutriments très appréciés aussi bien par l'Homme que par les micro-organismes saprophytes et pathogènes tels que les bactéries (Dadie et al., 2010; Ghazi et Niar, 2011). En général, on définit la qualité d'un produit comme étant l'ensemble des caractéristiques lui permettant de satisfaire les besoins exprimés par les consommateurs (Grenon, 2004). L'appréciation du lait peut passer par des caractéristiques comme l'analyse chimique et physique et aussi microbiologique. En effet, elles font partie des indicateurs de qualité. Dans la situation d'un pays Ouest Africain comme le Burkina Faso, pays à vocation pastorale avec un potentiel certain pour la production du lait, la maîtrise de la qualité est toujours en débat et des efforts continus sont nécessaires (Traoré et al., 2004 ; Millogo et al., 2010). L'augmentation rapide de la population et une urbanisation accélérée crée un besoin important en lait et produits laitiers et cette situation est similaire à celle de la plupart des pays Ouest Africains (Savadogo et Traoré, 2011 ; Corniaux, 2015). En effet, cette forte demande crée une importation massive de produits laitiers. Et, on se trouve là confronté à un dilemme, soit continuer à importer des produits laitiers ou soit mettre en œuvre des stratégies pour augmenter production au niveau national. L'option de produire a pris le dessus mais les produits laitiers locaux sont fortement concurrencés par ceux importés surtout dans un contexte où il existe de multiples circuits d'importation par la fraude qui échappent aux services de la douane et dégrade l'économie nationale. Cependant, la question de la qualité et de la compétitivité des produits locaux est soulevée et le consommateur n'est pas fixé sur son choix quand il veut acheter un produit laitier. Pour cela, dans le cadre du développement de la filière laitière bovine, un projet intitulé «Appui à l'amélioration durable de la productivité et de la compétitivité des filières laitières bovines en Afrique de l'Ouest et du Centre (AMPROLAIT)» a été conçu pour apporter des réponses à la productivité et à la compétitivité de la filière laitière bovine. Ainsi, il a été diagnostiqué que les différents acteurs de la chaîne laitière manquent de données suffisantes sur les paramètres du lait pour apprécier la qualité du lait afin de convaincre et rassurer les consommateurs. L'objectif de l'étude était d'analyser la qualité du lait aux différents maillons de la production, d'identifier les sources et le niveau de contamination bactérienne du lait et des produits laitiers et d'émettre des recommandations et proposer des kits d'application. Ainsi, les paramètres microbiologiques et physico-chimiques du lait et des produits le long de la chaîne 
permettent-ils d'apprécier la qualité du lait ? L'hypothèse est que l'existence de données sur la qualité des produits locaux peut rassurer les consommateurs et rendre ces produits plus compétitifs.

\section{MATERIEL ET METHODES}

\section{La zone d'étude et le choix des maillons de la chaîne de production du lait}

L'étude a été conduite dans la zone péri-urbaine de Bobo-Dioulasso de juillet à décembre 2013. Cette zone est définie comme étant l'espace situé à $50 \mathrm{~km}$ de rayon autour de la ville (Sidibé et al., 2004). Le choix de cette zone se justifie par le fait qu'elle est située dans la zone d'intervention du projet AMPROLAIT. La chaîne de production du lait comporte plusieurs maillons. Cependant, les points de collecte retenu sont la ferme, le collecteur, le vendeur ou la vendeuse au marché local, l'unité de transformation appelée laiterie et les supermarchés. Ce choix a été fait pour qu'à terme nous puissions proposer des voies d'amélioration de l'hygiène sous forme de kit. Un kit est un ensemble d'équipements couramment utilisés ou à utiliser par les acteurs de la chaîne de valeur lait et qui concourt à améliorer la qualité hygiénique afin de rendre les produits laitiers locaux compétitifs face aux produits laitiers importés.

\section{Echantillonnage des laits et produits laitiers}

Six fermes ont été sélectionnées dont cinq vaches par ferme. L'échantillon du seau vide était prélevé par récupération de l'eau de rinçage pour des analyses microbiologiques. Pour les échantillons de lait cru, le prélèvement se faisait directement au niveau des trayons pendant la traite. A la fin de la traite, un échantillon de lait était prélevé du seau de collecte représentant le lait de la traite totale. L'étude a concerné vingt collecteurs. Ils ont été sélectionnés de manière aléatoire selon les axes d'accès terrestre de la ville de Bobo-Dioulasso pour avoir un échantillonnage représentatif. Le laboratoire de recherche et d'enseignement en santé et biotechnologie animales (LARESBA) dispose d'un réseau d'unités de transformations du lait partenaires avec lesquelles il travaille dans le cadre de la plateforme d'innovation lait mise en place. Cinq laiteries ont été sélectionnées parmi celle-ci. Les vendeurs étaient ceux qui recevaient le lait pour le revendre. Vingt (20) vendeurs ont été retenus pour l'étude. Cinq (05) supermarchés installés à travers toute la ville ont été retenus. Les produits concernés dans notre échantillonnage étaient le lait pasteurisé et le yaourt. Tous les prélèvements de lait étaient divisés en deux aliquotes (analyse microbiologique et physicochimique). Ces prélèvements étaient conservés dans une glacière contenant des glaçons pendant le transport.

\section{Analyse des échantillons}

Le $\mathrm{pH}$ du lait a été mesuré à l'aide d'un pH-mètre (370 pH MeterJenway, Union Européenne). Les taux de matières grasses (MG), de matières protéiques (MP), de lactose (L), de matières sèches (MS) et de matières minérales $(\mathrm{MM})$ ont été déterminés à l'aide de la méthode infrarouge (Farm Milk Analyser 2001, Miris AB, Suède). Quatre groupes de microorganismes ont été étudiés : Escherichia Coli (Violet Red Bile Lactose Agar), la Flore Aérobie Mésophile Totale (Platte Count Agar), les Staphylococcus aureus (Baird Parker) et les Streptocoques (Bile Aesculin Azide Agar). Ce choix se justifie par leur capacité à provoquer des toxi-infections alimentaires pouvant créer des problèmes de santé publique (Kouame-Sina et al., 2010).

\section{Analyse statistiques}

Les analyses statistiques ont été effectuées à l'aide du logiciel statistique IBM 
SPSS Statistics 20. Les statistiques descriptives des moyennes ont été réalisées et le test de student a été utilisé pour comparer les moyennes et l'égalité des moyennes a été faite avec l'hypothèse des variances inégales. Les différences étaient considérées comme significatives au seuil de probabilité 0,05 . La corrélation de Pearson était très significative au seuil de 0,01 et significative au seuil de 0,05 .

\section{RESULTATS}

Le Tableau 1 montre que le taux de matières grasses obtenues pour le lait individuel par vache et celui des vendeuses présentent des valeurs assez faibles par rapport celui obtenu pour les laits de la traite totale des fermes, des collecteurs et des unités de transformation $(P<0,05)$. Pour le pourcentage de matières protéiques, de lactose, de matière sèche et de matières minérales, les laits individuels des vaches, les laits issus du mélange des fermes, des collecteurs et des unités de transformation ne présentent pas de différence significative. En revanche, comme dans le cas du pourcentage de matières grasses, le pourcentage de MP, de lactose, de MS et de MM est très faible pour les laits issus des vendeuses $(\mathrm{P}<0,05)$ avec les pourcentages obtenus pour les laits issus des autres maillons de la chaîne de production.
L'analyse de l'eau que les éleveurs utilisent pour nettoyer le matériel de traite et de conservation du lait a montré la présence de toutes les bactéries recherchées. Nous observons la présence de bactéries au niveau de tous les échantillons. Au niveau des vendeuses, les germes Escherichia coli et Streptocoques sont plus marqués. Les laits pasteurisés et les yaourts donnent des caractéristiques similaires en termes de contamination. Pour l'analyse microbiologique, la présence ou l'absence de bactérie a été retenue (Tableau 3). Les laits les plus chargés en microorganismes sont le lait des vendeurs, suivi de celui des collecteurs et du lait de la traite totale. Egalement, le récipient de collecte est très contaminé.

Les résultats des tests de corrélation de Pearson (Tableau 2) indiquent une corrélation positive très significative entre tous les composants physico-chimiques des laits sauf pour le $\mathrm{pH}$. Cependant, ces composants sont négativement corrélés par rapport à l'origine du lait. Les résultats montrent également que la présence de Escherichia coli dans un échantillon de lait, impliquait une présence des streptocoques et des staphylocoques (Tableau 4). Aussi, les streptocoques, les staphylocoques et la Flore Mésophile Aérobie Totale évoluent significativement en fonction de l'origine du lait (Tableau 4).

Tableau 1 : Composition moyennes des laits crus collectés au niveau des différents maillons de la chaîne de production.

\begin{tabular}{lcccccc}
\hline $\begin{array}{l}\text { Provenance } \\
\text { des laits }\end{array}$ & MG (\%) & MP (\%) & L (\%) & MS (\%) & MM (\%) & pH \\
\hline FVache & $2,3 \pm 1,4^{\mathrm{a}}$ & $3,8 \pm 0,4^{\mathrm{a}}$ & $5,2 \pm 0,3^{\mathrm{a}}$ & $12,1 \pm 2,3^{\mathrm{a}}$ & $9,7 \pm 0,9^{\mathrm{a}}$ & $6,6 \pm 0,1^{\mathrm{a}}$ \\
FMélange & $3,9 \pm 1,4^{\mathrm{b}}$ & $3,7 \pm 0,3^{\mathrm{a}}$ & $5,1 \pm 0,2^{\mathrm{a}}$ & $14,0 \pm 2,1^{\mathrm{b}}$ & $10,0 \pm 0,8^{\mathrm{a}}$ & $6,6 \pm 0,7^{\mathrm{a}}$ \\
Collecteurs & $4,4 \pm 0,5^{\mathrm{b}}$ & $3,7 \pm 0,2^{\mathrm{a}}$ & $5,2 \pm 0,1^{\mathrm{a}}$ & $14,6 \pm 0,7^{\mathrm{b}}$ & $10,2 \pm 0,3^{\mathrm{a}}$ & $6,4 \pm 0,1^{\mathrm{a}}$ \\
Laiteries & $4,2 \pm 0,6^{\mathrm{b}}$ & $3,5 \pm 0,2^{\mathrm{a}}$ & $5,2 \pm 0,2^{\mathrm{a}}$ & $14,2 \pm 0,8^{\mathrm{b}}$ & $9,9 \pm 0,3^{\mathrm{a}}$ & $6,5 \pm 0,0^{\mathrm{a}}$ \\
Vendeuses & $1,3 \pm 0,8^{\mathrm{c}}$ & $1,3 \pm 0,6^{\mathrm{b}}$ & $2,2 \pm 1,0^{\mathrm{b}}$ & $5,1 \pm 2,7^{\mathrm{c}}$ & $3,8 \pm 1,9^{\mathrm{b}}$ & $6,4 \pm 0,9^{\mathrm{a}}$ \\
\hline
\end{tabular}

Les valeurs ayant la même lettre en exposant ne sont pas statistiquement différentes au seuil de P > 0,05. F : Ferme ; Pourcentage de Matières grasses (MG); de Matières protéiques (MP); de Lactose (L); de Matières sèches (MS); de Matières minérales (MM). 
Tableau 2 : Corrélation des composants physico-chimiques.

\begin{tabular}{lccccccc}
\hline & Origine & MG & MP & L & MS & MM & PH \\
\hline Origine & - & $-0,003$ & $-0,000$ & $-0,000$ & $-0,000$ & $-0,000$ & NS \\
MG & 0,003 & - & 0,000 & 0,000 & 0,000 & 0,000 & NS \\
MP & $-0,000$ & 0,000 & - & 0,000 & 0,000 & 0,000 & NS \\
L & $-0,000$ & 0,000 & 0,000 & - & 0,000 & 0,000 & NS \\
MS & $-0,000$ & 0,000 & 0,000 & 0,000 & - & 0,000 & NS \\
MM & $-0,000$ & 0,000 & 0,000 & 0,000 & 0,000 & - & NS \\
PH & NS & NS & NS & NS & NS & NS & -
\end{tabular}

Pourcentage de Matières grasses (MG); de Matières protéiques (MP) ; de Lactose (L); de Matières sèches (MS) ; de Matières minérales (MM). La corrélation est significative au niveau 0,05 (bilatéral). La corrélation est significative au niveau 0,01 (bilatéral).

Tableau 3 : Présence ou absence des bactéries dans les seaux de collecte et des laits des différents maillons de la chaîne de production.

\begin{tabular}{lcccc}
\hline Provenance & Escherichia coli & $\begin{array}{c}\text { Flore Mésophile } \\
\text { Aérobie Totale }\end{array}$ & Streptococcus sp & $\begin{array}{c}\text { Staphylococcus } \\
\text { aureus }\end{array}$ \\
\hline F vache & $10,33 \pm 22,23 \mathrm{a}$ & $5,22 \pm 8,23 \mathrm{a}$ & $13,88 \pm 33,59 \mathrm{a}$ & $1,88 \pm 4,14 \mathrm{a}$ \\
F Mélange & $31,09 \pm 30,87 \mathrm{a}$ & $7,54 \pm 22,8 \mathrm{a}$ & $9,81 \pm 24,14 \mathrm{a}$ & $5,09 \pm 11,40 \mathrm{a}$ \\
F Seau & $30,00 \pm 49,71 \mathrm{a}$ & $8,66 \pm 16,47 \mathrm{a}$ & $10,22 \pm 26,23 \mathrm{a}$ & $1,89 \pm 4,14 \mathrm{a}$ \\
Collecteurs & $22,7 \pm 36,85 \mathrm{a}$ & $21,4 \pm 24,68 \mathrm{a}$ & $14,9 \pm 22,71 \mathrm{a}$ & $8 \pm 11,83 \mathrm{a}$ \\
Vendeuses & $57,8 \pm 42,34 \mathrm{a}$ & $3,3 \pm 8,81 \mathrm{a}$ & $51,3 \pm 46,73 \mathrm{a}$ & $3,4 \pm 6,74 \mathrm{a}$ \\
Past local & $39,40 \pm 23,7 \mathrm{a}$ & $64,80 \pm 131,73 \mathrm{c}$ & $2,4 \pm 2,61 \mathrm{~b}$ & $20,40 \pm 27,75 \mathrm{c}$ \\
Yaourt local & $13,43 \pm 35,29 \mathrm{a}$ & $93,28 \pm 141,43 \mathrm{~b}$ & $8,42 \pm 13,48 \mathrm{a}$ & $15 \pm 17,68 \mathrm{c}$ \\
\hline F: Ferme; Past : Lait pasteurisé. & & &
\end{tabular}

Tableau 4: Corrélation des composants microbiologiques.

\begin{tabular}{|c|c|c|c|c|c|}
\hline & origine & Escherichia coli & $\begin{array}{l}\text { Flore Mésophile } \\
\text { Aérobie Totale }\end{array}$ & Streptococcus sp & $\begin{array}{l}\text { Staphylococcus } \\
\text { aureus }\end{array}$ \\
\hline Origine & - & NS & 0,008 & NS & 0,027 \\
\hline Escherichia coli & NS & - & NS & 0,000 & 0,006 \\
\hline $\begin{array}{l}\text { Flore Mésophile } \\
\text { Aérobie Totale }\end{array}$ & 0,008 & NS & - & NS & NS \\
\hline Streptococcus sp & NS & 0,000 & NS & - & NS \\
\hline $\begin{array}{l}\text { Staphylococcus } \\
\text { aureus }\end{array}$ & 0,027 & 0,006 & NS & NS & - \\
\hline
\end{tabular}

La corrélation est significative au niveau 0,05 (bilatéral). La corrélation est significative au niveau 0,01 (bilatéral). 


\section{DISCUSSION}

Le lait est un aliment hautement nutritif de par sa richesse dont la composition dépend de son alimentation et d'autres facteurs intrinsèques (Kassa et al., 2016). On observe que le lait cru présente une composition chimique comparable aux données préalablement collectées dans la même localité au niveau des mêmes niveaux d'observation (Millogo et al., 2010). Cependant, on note une teneur en matières grasses et matières protéiques très faible (moins de 1,5\%) dans le lait des vendeurs et des vendeuses. Cela implique une mauvaise qualité physico-chimique du lait cru à ce point de vente. En plus le pH de ce lait est assez bas soit 6,47. En effet, le lait cru est une denrée fragile dont la finalité industrielle dépend de sa qualité. Au regard de ces résultats, on peut conclure que le lait cru des fermes, des collecteurs et des laiteries respecte les normes de lait cru. Par contre, le lait cru des vendeurs est impropre à la consommation humaine vu les valeurs de sa composition chimique. Ces valeurs obtenues dans le lait des vendeurs peuvent être dues au mouillage du lait. En effet, cette pratique est souvent effectuée par certains revendeurs. Il est ainsi impératif d'effectuer des sorties de contrôles dans les différents points de vente du lait. Selon une étude menée par Didnang et al. (2015), les laits pasteurisés locaux et les laits stérilisés ont des compositions chimiques similaires. Ces résultats sont aussi proches des valeurs obtenus dans le cadre des laits crus. Comme la stérilisation et la pasteurisation sont des techniques qui ont pour objectif l'élimination des germes pathogènes et le maintien de la valeur nutritive des laits, on peut dire que les laits pasteurisés par les laiteries dans la zone d'intervention du projet sont de bonne valeur nutritive si et seulement si il n'y a pas de contamination après le traitement thermique. Les yaourts fabriqués par les laiteries dans la zone du projet ont une valeur nutritive comparable à celle des yaourts importés (Didnang et al., 2015). Les observations des résultats montrent qu'on trouve des germes pathogènes dans les laits et les produits laitiers depuis la ferme jusqu'au niveau des supermarchés. En effet, la chaîne de froid quasi inexistante ne favorise pas une bonne conservation et la qualité du lait. Ainsi, l'exposition du lait à la température ambiante le long de la chaîne favorise l'altération de sa qualité (Kouamé-Sina et al., 2010). Pour le lait cru, la présence de ces germes s'explique par les conditions d'élevage (l'environnement de la traite, l'état sanitaire de l'animal, l'hygiène du trayon, ustensiles et l'eau utilisée) et le conditionnement au cours de la transaction du lait jusqu'à la laiterie (chaîne de froid et transport) (Aggad et al., 2009; Dadie et al., 2010 ; Kouamé-Sina et al., 2010). Ainsi, il n'est pas conseillé de consommer ce lait en l'état car la qualité est relativement non conforme. En effet, le risque d'infection existe pour les consommateurs et en particulier pour les enfants (Dadie et al., 2010 ). Cependant, pour les laits pasteurisés, stérilisés, la présence des germes s'explique par une récontamination des laits pendant le conditionnement comme nous l'avions précédemment observé dans une étude conduite par Sissao (2013). Ainsi, les laiteries doivent toujours garder à l'esprit que l'hygiène est primordiale tout au long de la chaîne de transformation du lait. La présence des mêmes germes dans le lait stérilisé peut être due à l'emballage. Les mêmes observations peuvent être faites sur les yaourts locaux et importés. Les données nous donnent un taux de la flore aérobie mésophile totale variable dans les laits crus et pasteurisés locaux. La présence de ces bactéries révèle un manque de respect des bonnes pratiques de production et de stockage. Les mêmes observations sont valables pour les yaourts. $\mathrm{La}$ 
présence des Staphylococcus aureus dans le lait est cependant très inquiétante. Cette bactérie dont la principale source est d'origine infectieuse (mammite subclinique) est présente dans $90 \%$ des fermes laitières (Kadja, 2010), En effet, si Staphylococcus aureus produit des enterotoxines, celles-ci seront importantes du point de vue de la technologie alimentaire étant donné qu'elles résistent à la chaleur (pasteurisation). Or, ces toxines causent des diarrhées et des vomissements importants (Faye et Loiseau, 2002 ; Barkema et al., 2006 ; Jakob et al., 2011). Egalement, la présence de Escherichia coli dans ses produits est grave du fait de sa pathogénicité qui est la première cause de diarrhée chez les enfants (Donnenberg, 2005 in Dadie et al., 2010). Ainsi, il est impératif de traiter convenablement le lait cru avant toute consommation. On peut dire, en conclusion, qu'il existe un problème d'hygiène du lait au niveau des différents maillons de la chaîne de production au Burkina Faso comme celui observé par Kouamé-Sina et al. (2010) sur le lait cru à la ferme et dans les points de vente.

Les présents résultats (Tableau 1 et Tableau 3) et ceux obtenus dans les publications antérieures que nous avions produites (Millogo et al., 2010; Millogo et al., 2014 ; Didnang et al., 2015 ; Sissao et al., 2015) permettent de dire que pour maitriser l'hygiène du lait local, il faut des Kits d'hygiène à la ferme, au niveau du collecteur et de la laiterie. Ceci devrait permettre d'amoindrir le niveau de contamination des laits, d'améliorer l'hygiène du lait de consommation et ainsi de rendre le lait local compétitif.

\section{Conclusion}

Cette étude a permis de confirmer certaines inquiétudes qui freinent la compétitivité des produits laitiers locaux au Burkina Faso. En effet, la question d'hygiène des produits est la principale préoccupation des consommateurs de lait. Les résultats montrent une bonne qualité physico-chimique des laits aux différents maillons de la chaîne sauf au niveau du maillon vendeuse locale, ce qui donne une idée de la bonne valeur nutritive des produits laitiers. La présence des bactéries observées est soit due aux différents points de contamination soit à une récontamination au niveau du conditionnement. Il est donc impératif et important de contrôler rigoureusement la qualité physico-chimique, hygiénique de production et de transformation du lait mis à la disposition des populations. A cet effet, un effort supplémentaire des acteurs de la filière est nécessaire. C'est pourquoi, en recommandation, des kits d'hygiène simples et accessibles sont proposés. Cela pourrait contribuer à améliorer la qualité hygiénique de la production nationale au niveau des maillons critiques, chose qui pourrait rassurer le consommateur local.

\section{CONFLIT D'INTERETS}

Les auteurs déclarent ne pas avoir de conflits d'intérêts en relation avec cet article.

\section{CONTRIBUTIONS DES AUTEURS}

VM a validé le protocole expérimental, a été responsable de l'expérimentation, de l'exploitation des résultats et de la rédaction du manuscrit. Il a été le responsable scientifique de cette étude. MS a participé à la rédaction du manuscrit. GAO a contribué à l'amélioration du manuscrit et responsable du laboratoire où se tient les travaux.

\section{REFERENCES}

Aggad H, Mahouz F, Ahmed Ammar Y, Kihal M. 2009. Evaluation de la qualité hygiénique du lait dans l'Ouest Algérien. Revue Med. Vet., 160(12): 590-595.

Barkema HW, Schukken YH, Zadoks RN. 2006. Invited Review: The role of cow, 
pathogen, and treatment regimen in the therapeutic success of bovine Staphylococcus aureus mastitis. J. Dairy Sci., 89(6): 1877-1895. DOI : 10.3168/jds.S0022-0302(06)72256-1.

Corniaux C. 2015. L'industrie laitière en Afrique de l'Ouest: histoire, stratégie et perspectives. Projet "milky way for development”. CIRAD/PPZS. 39 p. https://agritrop.cirad.fr/document_57531 1.pdf. Consulté le 20/08/2017.

Dadie A, Nzebo D, Guessennd N, Dako E, Dosso M. 2010. Prévalence de Escherichia coli entéropathogènes dans le lait non pasteurisé produit à Abidjan, Côte d'Ivoire. Int. J. Biol. Chem. Sci., 4(1): 11-18. http://ajol.info/index.php/ijbcs

Didnang C, Millogo V, Molele FM, Ouedraogo GA. 2015. Evaluation de la qualité nutritive des laits pasteurisés et des yaourts fabriqués au Burkina Faso. Afrique Science, 11(1): 155 - 166. ISSN 1813-548X, http://www.afriquescience. info.

Donnenberg MS. 2005. Enterobacteriaceae. In Principles and Practice of Infectious Diseases, Mandell GL, Bennett JE, Dolin R, Mandell, Douglas, Bennett's (eds). Elsevier Churchill Livingstone 2: Philadelphia; 2567-2586.

Faye B, Loiseau G. 2002. Sources de contamination dans les filières laitières et exemples de démarches qualité. In Gestion de la Sécurité des Aliments dans les Pays en Développement. Actes de l'Atelier International, Hanak E, Boutrif E, Fabre P, Pineiro M (eds). CIRAD-FAO, 11-13 décembre 2000, CIRAD-FAO. Cédérom du CIRAD : Montpellier, France. 5 p.

Ghazi K, Niar A. 2011. Qualité hygiénique du lait cru de vache dans les différents élevages de la Wilaya de Tiaret (Algérie). Tropicultura, 29(4): 193-196.
Grenon C. 2004. Lait de Qualité. Symposium sur les bovins laitiers. Centre de Référence en Agriculture et Agroalimentaire du Québec. 33 pages. https://www.agrireseau.net/bovinslaitiers /documents/Grenon_Claude.pdf Consulté le 09/02/2018.

Jakob E, Winkler H, Schaeren W, Amrein R, Geinoz M. 2011. La qualité du lait cru, un défi permanent. Edition Agroscope Liebefeld-Posieux forum, 78: 5-17.

Kadja MC. 2010. Etude des mammites subcliniques dans les élevages bovins laitiers en Afrique de l'Ouest, cas du Sénégal et du Bénin. Thèse de doctorat Université Cheikh Anta Diop de Dakar. 156 pages.

Kassa KS, Ahounou S, Dayo GK, Salifou C, Issifou MT, Dotché I, Gandonou PS, Yapi-Gnaoré V, Koutinhouin B, Mensah GA, Youssao IAK. 2016. Performances de production laitière des races bovines de l'Afrique de l'Ouest. Int. J. Biol. Chem. Sci., 10(5): 2316-2330. DOI : http://dx.doi.org/10.4314/ijbcs.v10i5.29

Kouamé-Sina SM, Bassa A, Dadie A, Makita K, Dje M, Bonfoh B. 2010. Analyse des risques microbiens du lait cru local à Abidjan (Côte d'Ivoire). Revue Africaine de Santé et de Productions Animales, 8(5): $8 \mathrm{p}$.

Millogo V, Sissao M, Sidibe/Anago AG, Ouédraogo GA. 2015. Effect of storage time and temperature on raw milk composition of dairy cattle in tropical conditions. African Journal of Dairy Farming and Milk Production, 2(1): 104-108

Millogo V, Svennersten Sjaunja K, Ouédraogo GA, Agenäs S. 2010. Raw milk Hygiene at farms, processing units and local markets in Burkina Faso. Food Control.,

21: 1070-1074. 
https://doi.org/10.1016/j.foodcont.2009.1 2.029

Savadogo A, Traore AS. 2011. La flore microbienne et les propriétés fonctionnelles des yaourts et laits fermentés. Int. J. Biol. Chem. Sci., 5(5): 2057-2075.

DOI:

http://dx.doi.org/10.4314/ijbcs.v5i5.28

Sidibé M, Boly H, Lakouetene T, Leroy P, Bosma RH. 2004. Characteristics of periurban dairy herds of Bobo-Dioulasso, Burkina Faso. Tropical Animal Health and Production, 36: 95-100.

Sissao M, Millogo V, Ouédraogo GA. 2015. Composition chimique et qualité bactériologique des laits crus et pasteurisés au Burkina Faso. Afrique Science, 11(1): 142- 154.
Sissao M. 2013. Analyse de la composition du lait de la ferme et de la laiterie en fonction du taux de Staphylococcus aureus, de Escherichia coli et de cellules somatiques de la zone de BoboDioulasso. Mémoire de Master de Biologie appliquée et Modélisation des Systèmes Biologiques. Institut du Développement Rural/Université Polytechnique de Bobo-Dioulasso. 87 pages.

Traoré A, Tamboura HH, Bayala B, Rouamba DW, Yameogo N. 2004. Prévalence globale de pathologies majeures liées à la production laitière bovine en système d'élevage intra urbain à Hamdallaye (Ouagadougou). Biotechnol. Agron. Soc. Environ., 8(1): 3-8. 